\title{
A PROSPECTIVE STUDY ON CLINICAL PROFILE, DIAGNOSIS AND MANAGEMENT OF BLUNT TRAUMA ABDOMEN IN A TERTIARY CARE HOSPITAL
}

\section{Kaushik Mandal}

Anirban Bhunia*

\author{
Associate Professor, Department of General Surgery, Burdwan Medical \\ College (BMC)
}

Junior Resident, Department of General Surgery, Burdwan Medical College (BMC). *Corresponding Author

\section{ABSTRACT}

Blunt abdominal trauma (BAT) is one of the most common injuries most often results from a motor vehicle collision and such incidents, combined with pedestrian versus automobile collisions. AIMS: To evaluate the demographic and clinical profile, diagnosis and management of blunt abdominal trauma METHODS: Our study was conducted over a period of 1 year (April 2019 to march 2020). A total no of 107 patients fulfilling inclusion criteria are allotted in the study and those with severe head injuries, vascular injuries and with major comorbidities are excluded from the study. RESULTS: Males are predominantly affected. It is mostly seen in the age group of 21-40 yrs which form the young and reproductive group. Road traffic accidents form the most common mode of injury. FAST is a very important investigation. The most commonly injured organ in present study is liver which is managed by hepatorraphy and absorbable gelatin sponge packing. The present study showed a mortality rate of $6.5 \%$.

\section{KEYWORDS : Blunt abdominal trauma, Focused Assessment with Sonography for Trauma (FAST)}

\section{INTRODUCTION}

Blunt abdominal trauma (BAT) is one of the most common injuries most often results from a motor vehicle collision and such incidents, combined with pedestrian versus automobile collisions, account for over 75 percent of cases. Blows to the abdomen e.g. assault with blunt objects, sport injuries, industrial mishaps, bomb blast injuries and falls are responsible for 15 and 6-9 percent, respectively ${ }^{[1]}$. The prevalence of intra-abdominal injury among patients presenting to the emergency department with BAT is approximately $13 \% .{ }^{[2]}$

The rapid increase in motor vehicles and its aftermath has caused rapid increase in number of victims to blunt abdominal trauma. Blunt abdominal trauma is the leading cause of morbidity and mortality among all age groups. Identification of serious intra-abdominal pathology is often challenging. Many injuries may not manifest during initial assessment and treatment period. Missed intra-abdominal injuries and concealed haemorrhage are frequent causes of increased morbidity and mortality, especially in patients who survive the initial phase after an injury.

Evaluating the abdomen in blunt trauma remains a clinical challenge. Physical examination may be misleading when there are proximity injuries such as lower rib fracture or pelvic fracture. Injuries may be missed by physical examinations when there is altered mental status from drugs, alcohol, or associated head trauma. The physical examination is unreliable when there is associated spinal cord injury. Using vital signs as a guide to intra-abdominal injuries is also unreliable, since haemorrhage may be from other sites and stable vital signs can be associated with an intra-abdominal injury.

Most commonly involved organs in blunt trauma abdomen are solid organs like spleen, liver, kidney and retro-peritoneum while hollow viscus injuries are relatively rare. ${ }^{[3,4]}$

In recent years, Focused Assessment with Sonography for Trauma (FAST) has emerged as a useful diagnostic test in the evaluation of BAT. FAST is non-invasive, may be easily performed, and can be performed concurrently with resuscitation. Routine use of CT scanning for the evaluation of BAT was not initially viewed with overwhelming enthusiasm. ${ }^{[5]}$ CT scanning requires a cooperative, hemodynamically stable patient. Due to inadequate treatment of abdominal injuries, outcome is fatal in most of the cases. The knowledge in the management of blunt abdominal trauma has progressively increasing due to the in-patient data gathered from different parts of the world. In spite of the best techniques and advances in diagnostic and supportive care, the morbidity and mortality remains at large. The reason for this could be due to the interval between trauma and hospitalization, delay in diagnosis, inadequate and lack of appropriate surgical treatment, post-operative complications and associated trauma especially to head, thorax and extremities.

\section{METHODOLOGY}

\section{Aims and objectives}

1. To evaluate the patients with blunt abdominal trauma with respect to demographic profile (i.e. Age, Sex, Occupation, BMI, Mechanism of trauma etc.).

2. To study the Clinical presentation (Symptoms, Clinical Signs, Vital Parameters, Systemic examination etc.).

3. To evaluate the various diagnostic modalities including Focused Assessment with Sonography for Trauma (FAST) and Computed Tomography of Abdomen.

4. To evaluate the treatment modalities and management.

All consenting Blunt Abdominal Trauma Cases in between April 2019 to March 2020 admitted in all surgical units of Burdwan Medical College and Hospital was considered. A total no of 107 patients fulfilling inclusion criteria are allotted in the study and those with severe head injuries, vascular injuries and with major comorbidities are excluded from the study.

Data will be collected from indoor patient records, operation theatre records and outpatient records of the study cases in a preformed Performa. Permission from Institutional Ethics Committee was taken. Written informed consent was obtained from all participating patients.

Statistical analysis plan

- Standard statistical methods for data compilation and analysis.

- Software package like SPSS(2015) was applied for statistical analysis

\section{RESULT}

In this series, the majority of the patients belonged to $21-40$ yrs.

In the 107 cases studied, 91 were male and 16 were females, 
thus making majority of male patients.

In this case series majority of patients have BMI of range 19-25, with very less patients outside the range.

After a detailed clinical evaluation and suitable investigations, out of 48 patients of hemoperitoneum, 40 patients underwent exploratory laparotomy because of hemodynamic instability and 8 were considered for conservative management as they are hemodynamically stable. All of 17 patients of perforative peritonitis underwent laparotomy

Road traffic accidents were responsible for $57 \%$ of blunt abdominal trauma cases while fall from height accounted for $28 \%$ of cases and blow to abdomen with blunt objects were responsible for $15 \%$ of cases.

Majority of victims of blunt abdominal trauma were labourers and agricultural workers accounting for $44.9 \%$ whereas retired persons and housewives suffered least.

Majority of patients presented with abdominal pain (85.9\%) and tenderness (80.4\%).

\begin{tabular}{|c|c|c|}
\hline Features & No. of patients & $\%$ \\
\hline Free gas under diaphragm & 7 & $6.5 \%$ \\
\hline Ground glass appearance & 5 & $4.7 \%$ \\
\hline No abnormality detected (NAD) & 62 & $57.9 \%$ \\
\hline Not done & 33 & $30.8 \%$ \\
\hline
\end{tabular}

The above table shows the abnormal finding detected in X-ray erect abdomen and their percentage. Plain X-ray abdomen was done in 74 cases, out of total 107 cases. This was not done in 33 patients as the condition of patient did not permit to shift them to X-ray room or they died while being resuscitated for shock.

Free gas under diaphragm was found in 7 cases out of 20 bowel perforation cases found at laparotomy.

Four quadrant aspirations were done in 72 cases, among which 48 were positive and 24 were negative. Out of 24 negative cases, 7 were false negative. On laparotomy they were found to have hemoperitoneum.

Focussed Assessment with Sonography for trauma (fast) scan showed liver injury in $29.9 \%$, splenic injury in $28 \%$ and collection in the peritoneal cavity in $26.2 \%$ cases.

Latent period is interval between the time of injury and the time of surgery. Average latent period seen in present study is between 9-16 hrs. Majority of patients were taken for surgery between 9-12 hrs of latent period in this study.

In this series liver (35.5\%) is the most common organ injured followed by spleen (28.9\%) and small bowel (12.2\%).

\begin{tabular}{|c|c|c|}
\hline Organs injured & No. of patients & $\%$ \\
\hline Liver & 38 & 35.5 \\
\hline Spleen & 31 & 28.9 \\
\hline Mesentery & 12 & 11.2 \\
\hline $\begin{array}{c}\text { Small intestine } \\
\text { (jejunum/ileum) }\end{array}$ & $4+9=13$ & 12.2 \\
\hline Retroperitoneum & 8 & 7.5 \\
\hline Kidney & 9 & 8.4 \\
\hline Bladder & 1 & 0.9 \\
\hline Pancreas & 3 & 2.8 \\
\hline Duodenum & 3 & 2.8 \\
\hline Colon & 2 & 1.9 \\
\hline Stomach & 2 & 1.9 \\
\hline
\end{tabular}

In this study out of 38 liver injury patients, 20 needs laparotomy and hepatorraphy \& absorbable gelatin sponge packing done while 18 patients of liver injury were managed conservatively. 18 patients need splenectomy and 3 needs splenorrhaphy out of 31 patients while 10 patients were managed conservatively. Bowel perforations were treated with 2 layered closures, with only l patient requiring resection \& anastomosis. Omental and mesenteric injuries were treated by simple suturing and ligating the bleeding points. 2 patients need nephrectomy due to expanding retroperitonal hematoma. Above data shows splenectomy is required in $16.8 \%$, splenorrhaphy in $1.9 \%$, liver injury repair in $18.7 \%$, mesenteric repair in 5.6\%, intestinal injury repair in $14 \%$.

\begin{tabular}{|c|c|c|c|}
\hline Procedure & $\begin{array}{c}\text { No. of } \\
\text { patients }\end{array}$ & $\%$ & $\begin{array}{c}\text { Total no of patients of } \\
\text { respective organ injury }\end{array}$ \\
\hline Splenectomy & 18 & 16.8 & 31 \\
\hline Splenorraphy & 3 & 2.8 & 31 \\
\hline Liver repair & 20 & 18.7 & 38 \\
\hline Bladder repair & 1 & 0.9 & 1 \\
\hline Nephrectomy & 2 & 1.9 & 9 \\
\hline Mesentery repair & 6 & 5.6 & 12 \\
\hline $\begin{array}{c}\text { Repair of intestinal } \\
\text { injury }\end{array}$ & 15 & 14.0 & 15 \\
\hline Stomach repair & 2 & 1.9 & 2 \\
\hline Duodenum repair & 3 & 2.8 & 3 \\
\hline
\end{tabular}

Most common complication following operation is SSI (14\%) followed by RTI (12.2\%). UTI and wound dehiscence account for $7.5 \%$ and $2.8 \%$ respectively.

The average range of stay of patients in the hospital is 8-15 days while range varies from 4-35 days.

A total of 7 patients died in this study. 4 patient died during resuscitation and 3 patient died in postoperative period due to peritonitis and septicaemia. None of patients died who were put for conservative management after proper investigations. The mortality in present study is $6.5 \%$.

\section{DISCUSSION}

It can be seen from above table that the majority of patients belonged to 21-40 yrs of age group. In Davis et al and Khanna et al studies the majority of patients belonged to 21-30 yrs age group. Therefore it can be concluded that the young and the productive age group are the usual victims of blunt abdominal trauma.

The above table shows that there is an increasing trend towards conservative management. In present study $39 \%$ of patients were subjected for non-operative management. Davis et al showed $23 \%$ and Khanna et al showed that $42 \%$ of patients were subjected for conservative management. Conservative management is gaining increasing acceptance mainly because of easy availability of FAST and CT scan. With the aid of CT scan it is possible to accurately grade the extent of injury to solid organs like spleen and liver. Minor lacerations and capsular tear, difficult to diagnose clinically can be demonstrated by CT scan and selected for conservative management. The disadvantages of conservative management are those of missed injuries and delayed treatment resulting in excessive morbidity and even mortality.

The above table clearly depicts that road traffic accidents is the most common mode of injury. This is due to the rapid development in technology in all fields including automobile industry where the first priority is given to speed rather than safety.

In the present study, abdominal pain was the most common presenting complains (86\%) and abdominal tenderness was the most common sign (80\%). But the signs and symptoms are 
notoriously unreliable and are often masked by concomitant head injury, chest injury, and pelvic fractures. Significant injury to retro peritoneum may not manifest signs and symptoms immediately and be totally missed even on abdominal X-ray and FAST predisposing to grave consequences of missed injuries. In Davis et al study, $43 \%$ patients had no specific complaints and no signs and symptoms of intra-abdominal injury when first presented to emergency room. But $44 \%$ of those patients eventually required exploratory laparotomy and $34 \%$ had an intraabdominal injury. This emphasizes the importance of careful and continuing observation and repeated examination of individuals with abdominal trauma.

Latent period is interval between the times of injury to the time of surgery. $54 \%$ of patients were taken for surgery between 9$16 \mathrm{hrs}$ and $14 \%$ patients between 5-8 hrs of injury. This time lag is due to site of accidents, which are usually rural, and the time taken to transport them to the hospital. Few patients were taken for surgery after $24 \mathrm{hrs}$ as they were initially put for conservative management. Since their condition deteriorated on repeated clinical examination, they had to be taken up for delayed exploratory laparotomy.

Plain x-ray abdomen was done in 84 cases, out of the total 107 cases. Free gas under diaphragm was found in 7 cases out of 20 cases of bowel perforations detected at laparotomy. So the sensitivity of plain X-ray abdomen in detecting pneumoperitoneum is $35 \%$ in present study. Davis et al reported that in their series, abdominal X-ray was abnormal in $21 \%$ of cases; pneumoperitoneum was detected in $6 \%$ of cases and dilated bowel loops in $6 \%$ of cases.

In the present study $67 \%$ of patients were subjected to four quadrant aspirations as against $44 \%$ in Davis et al study. 48 cases were found to positive and 24 cases were negative. Out of these 24 cases, 7 were false negative in this study. The sensitivity of this investigation in present study is $87.5 \%$. Correct results (positive/negative), as determined by subsequent laparotomy, were obtained in $86 \%$ of cases in Davis et al study ${ }^{[1]}$

DPL was not done in this study owing to its complication rates.

All patients were subjected to FAST excepting 4 patients who died during resuscitation. 6 patients having grade I liver or splenic injury or pancreatic injury were labelled as normal while in others FAST was correctly able to detect solid organ injury. Therefore FAST is more reliable in detecting solid organ injuries and free fluid in abdomen. In Yoshi et al study, the sensitivity of ultrasound in detecting injuries in blunt trauma abdomen patients is about $94.6 \%$. ${ }^{[5] 1]}$

The above table compares the incidence of the organs involved in blunt abdominal trauma in present study to that of the international series. In our study most common organ involved is liver while in others most common organ involved is spleen. Small bowel injury accounts for minor percentage of patients which is quite comparable to other studies.

In the present study closure of bowel perforation was done in 15 patients, stomach perforation closure in 2 while duodenal perforation repair done in 3 patients. Splenectomy and splenorrhaphy in $18 \& 3$ patients respectively while liver repair in 20 patients. Nephrectomy was done in 2 patients while lcase of bladder rupture was repaired. Rests of the cases were managed conservatively.

A total of 7 patients died in this study. 4 patient died during resuscitation and 3 patient died in postoperative period due to peritonitis and septicaemia. None of patients died who were put for conservative management after proper investigations.
The mortality in present study is $6.5 \%$. In other studies like Davis et al, Di Vincenti et al, Cox et al mortality rates are $13.3 \%, 23 \%, 10 \%$ respectively which are high as compared to our study due exclusion of patients of simultaneous head injury or chest injury.

\section{CONCLUSION}

This was a prospective study of 107 cases of blunt abdominal trauma in Burdwan Medical College and Hospital, Burdwan from April 2019 to March 2020. From this study, the following conclusions can be made:

- Males are predominantly affected. It is mostly seen in the age group of 21-40 yrs which form the young and reproductive group. These patients are usually from lower socioeconomic income group.

- Road traffic accidents form the most common mode of injury. Hence measures should be taken to prevent these accidents and care of victims at accident site. Well established trauma care centers should be established at least every district hospital. Measures for early transport of patients from accident site to trauma center should be undertaken.

- A thorough and repeated clinical examination and appropriate diagnostic investigations lead to successful treatment in these patients.

- Though conservative management is successful in carefully selected patients, operative measures remain the main stay of treatment.

- Plain X-ray abdomen is a valuable investigation taken for gastrointestinal injuries.

- FAST is a very important investigation which gives a very clear picture of solid organ injury and free fluid in the peritoneal cavity.

- Four quadrant aspiration is a simple and important tool for diagnosis with $>90 \%$ sensitivity.

- The most commonly injured organ in present study is liver which is managed by hepatorraphy and absorbable gelatin sponge packing.

- 2nd most common organ injured is spleen, majority of which is treated with splenectomy and few cases splenorrhaphy.

- Small bowel injuries come in 3rd positions which are mainly managed by primary repair.

- Retroperitoneal injuries were seen in small proportion of patients associated with renal injury. Most of them were managed by conservatively. Only renal pedical trauma or expanding retroperitoneal hematoma needs exploration.

- Post-operative complications like SSI, RTI, UTI, wound dehiscence and fistula are common in blunt abdominal trauma.

- The present study showed a mortality rate of $6.5 \%$.

\section{REFERENCES}

1. Davis JJ, Cohn I Jr, Nance FC. Diagnosis and management of blunt abdominal trauma. Ann Surg 1976; 183:672.

2. Nishijima DK, Simel DL, Wisner DH, Holmes JF. Does this adult patient have a blunt intra-abdominal injury? JAMA 2012; 307:1517.

3. Cornelis H van der Vlies, Dominique C Olthof, Menno Gaakeer, Kees J Ponsen, Otto M van Delden and J Carel Goslings; Changing patterns in diagnostic strategies and the treatment of blunt injury to solid abdominal Organs; van der Vlies et al. International Journal of Emergency Medicine 201 1, 4:47.

4. Hoff et al. EAST Practice Management Guidelines Work Group Practice Management Guidelines for the Evaluation of Blunt Abdominal Traum,2001. www.east.org.

5. Matsubara TK, Fong HM, Burns CM. Computed tomography of abdomen (CTA) in management of blunt abdominal trauma. J Trauma. 1990;30:410-414. 